accompanied by dark lines on their more refrangible sides."

This was substantially the substance of the telegram which appeared in the Standard on the following Wednesday (February 10), with the additional remark that the Harvard astronomers thought it possible that the phenomena presented by the new star had been caused by the collision of two celestial bodies.

On the next day the detailed observations made on Sunday night at Kensington, together with the approximate wave-lengths of the lines measured on the photographs, were sent on by Mr. Lockyer to the Royal Society. From these we learn that the Nova on Sunday appeared to be slightly brighter than on February 3 .

With the Io-inch refractor and Maclean spectroscope, $\mathrm{C}$ was seen to be very brilliant, and there were four very conspicuous lines in the green. Several fainter lines were also seen, and a dark line was suspected in the orange. Mr. Lockyer noticed that some of the lines, especially the bright one near F, on the less refrangible side, appeared to change rapidly in relative brightness, and this was confirmed by Mr. Fowler.

Observations of the spectrum were made by Mr. Fowler with the 3-foot reflector and the Hilger 3-prism spectroscope. These call for no special remark.

Twenty bright lines have been measured on the photographs, and their wave-lengths are given in the accompanying table:-

\begin{tabular}{|c|c|c|c|c|c|c|}
\hline \multicolumn{4}{|c|}{ Lines in the spectrum of Nova Aurig:e. } & \multirow[b]{2}{*}{ 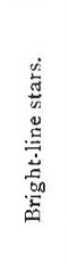 } & \multirow[b]{2}{*}{ 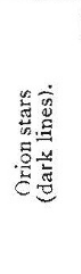 } & \multirow[b]{2}{*}{ 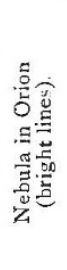 } \\
\hline 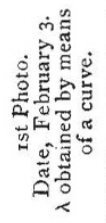 & 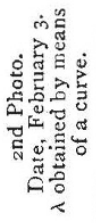 & 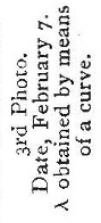 & 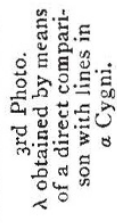 & & & \\
\hline K 3933 & 3933 & 3933 & 3933 & - & 3933 & 3933 \\
\hline H 3968 & 3968 & 3968 & 3968 & 3970 & 3968 & 3968 \\
\hline h 4IOI & $4 \mathrm{IOI}$ & $4 \mathrm{IOI}$ & $410 I$ & 4101 & $4 \mathrm{IOI}$ & $4 \mathrm{IOI}$ \\
\hline 4128 & 4130 & 4127 & 4128 & - & 4130 & 4130 \\
\hline 4172 & 4172 & 4172 & 4172 & 一 & $4^{172}$ & - \\
\hline & - & - & 4202 & 4200 & - & 4200 \\
\hline 4226 & 4227 & 4228 & 4226 & - & - & 4226 \\
\hline 4268 & 4268 & - & 4264 & - & 4268 & 4268 \\
\hline - & - & 4294 & $429 i$ & - & - & - \\
\hline 4312 & 43 IO & 4310 & 4310 & - & - & - \\
\hline G 4340 & $434^{\circ}$ & 4340 & 4340 & 4340 & 4340 & 4340 \\
\hline - & - & - & $\begin{array}{l}4383 \\
4412\end{array}$ & - & 4415 & 4383 \\
\hline - & 二 & - & $\begin{array}{l}4412 \\
4434\end{array}$ & - & $\underline{445}$ & 4410 \\
\hline - & - & - & 4469 & 4472 & 4472 & 4472 \\
\hline 4516 & 4516 & 4522 & 4518 & $45 \mathrm{IO}$ & & \\
\hline 4552 & $455^{2}$ & 4554 & 4555 & $455^{\circ}$ & - & - \\
\hline $45^{8} 7$ & $45^{87}$ & 4584 & 4587 & - & 一 & - \\
\hline 4618 & 4618 & 4625 & 4625 & 4620 & - & - \\
\hline - & - & 4860 & $4860 \mathrm{~F}$ & 4860 & 4860 & 4860 \\
\hline
\end{tabular}

The table also shows probable coincidences with the lines in the spectra of the Wolf-Rayet stars as photographed by Prof. Pickering, dark lines in Orion stars photographed at Kensington, and bright lines in the Orion nebula photographed at Mr. Lockyer's observatory at Westgate.

In addition to the lines recorded in the table, the photographs in the spectrum of the Nova show several lines more refrangible than $\mathrm{K}$. They probably include some of the ultra-violet hydrogen lines.

All the lines in the spectrum of the Nova are broad, although in a photograph of the spectrum of Arcturus, taken with the same instrumental conditions, the lines were perfectly sharp. It is also important to note that the broadening of the lines is not accompanied by any falling off of intensity at the edges, as in the case of the hydrogen lines in such a star as Sirius. With the method employed in taking the photographs, long exposures are liable to result in a thickening of all the lines on account of atmospheric tremors. The lines would also be thick if the Nova be hazy. In the photograph, however, all the lines are not equally thick.

If the lines are similarly broadened when a slit spectroscope is employed, the effect must be due to internal agitations, for if different regions of the Nova are moving with varying velocity, or with the same velocity in different directions, a normally fine line might be widened in the manner observed in the photographs.

With regard to the bright and dark lines the paper states as follows:-

"A somewhat similar phenomenon has already been recorded by Prof. Pickering in the case of $\beta$ Lyre, and this has been confirmed by a series of photographs taken at Kensington. In this case the bright lines are alternately more or less refrangible than the dark ones, with a period probably corresponding to the known period of variation in the light of the star. The maximum relative velocity indicated is stated by Prof. Pickering as approximately 300 English miles per second.

"In the case of Nova Aurigæ, the dark lines in all four photographs taken at Kensington are more refrangible than the bright ones, so that as yet there is no evidence of revolution.

"The relative velocity indicated by the displacement of the dark lines with respect to the bright ones appears to be over rather than under 500 miles per second. The reduction is not yet complete.

"Should the photographs which may be obtained in the future continue to show the dark lines displaced to the more refrangible side of the bright ones, it will be a valuable confirmation of my hypothesis as to the causes which produce a new star-namely, the collision of two meteor-swarms On this supposition the spectrum of Nova Aurigæ would suggest that a moderately dense swarm is now moving towards the earth with a great velocity and is disturbed by a sparser one which is receding. The great agitations set up in the dense swarm would produce the dark-line spectrum, while the sparser swarm would give the bright lines."

\section{ELECTRODYNAMIC THEORIES AND THE ELECTROMAGNETIC THEORY OF LIGHT:}

$\mathrm{I}$ a former article we endeavoured to give an account of the first part of M. Poincaré's "Électricité et Optique," in which he dealt with the electric and magnetic theories expounded in Maxwell's treatise. In Part II. he now compares the theory of electromagnetic action given by Maxwell with the somewhat more general theory put forward by Helmholtz in his celebrated paper on the equations of motion of electricity (Pogg. Ann., cii. p. 529, or Wissensch. Abhand., vol. i.) ; discusses the condition which must hold in order that the two theories may coincide ; and, after a masterly exposition of the various consequences which flow from Maxwell's theory, finishes with a very valuable analysis of the theoretical and experimental work of Hertz.

In the first chapter M. Poincaré deals with the formula of Ampère for the mutual action of two current elements. The method adopted is founded on the following three principles assumed from Ampère's experiments :--

(I) That a current in a conductor may be replaced by an equal current in a sinuous conductor nowhere deviating from the first by a distance comparable with the distance of the latter from any element of the other conductor acted upon.

x " Électricité et Optique." II. Les 'I'héories de Helmholtz et les Expériences de Hertz. Par H. Poincaré, Membre de l'Institut. (Paris : Georges carré, r8gr.)

NO. I I 64, VOL. 45] 
(2) The action of a closed circuit carrying a current upon any current element is normal to the element.

(3) The action of a closed (non-varying) solenoid upon a current element is zero.

It is besides assumed that the action of a circuit upon a current element is the sum, in the dynamical sense, of the individual actions of the elements of the circuit; and that the action between two elements is a force in the straight line joining their centres.

The process used for the deduction of Ampère's formula from these premisses is very elegant. If $d s, d s^{\prime}$ be the lengths of the two elements, $\gamma, \gamma^{\prime}$ the currents in them, $\epsilon$ the angle between the elements, $\theta, \theta^{\prime}$ the angles they make with the line joining their centres, the action of $d s$ on $d s^{\prime}$ may be represented by $f\left(r, \theta, \theta^{\prime}, \epsilon\right) \gamma \gamma^{\prime} d s d s^{\prime}$. But the action of $d s$ may, by the first principle stated above, be replaced by the actions, of its components $d x, d y, d z$; so that

$$
f=\mathrm{A} \frac{d x}{d s}+\mathrm{B} \frac{d y}{d s}+\mathrm{C} \frac{d z}{d s},
$$

where $\mathrm{A}, \mathrm{B}, \mathrm{C}$ are coefficients. Now, $f$ depends upon $r, \theta, \theta^{\prime}, \epsilon ; r$ and $\theta^{\prime}$ do not depend on the direction cosines of $d s ; \cos \theta$ and $\cos \epsilon$ are linear and homogeneous with respect to these direction-cosines. Hence $f$ must be linear and homogeneous with respect to $\cos \theta$ and $\cos \epsilon$, that is with respect to $d r / d s$, and $d^{2} r / d s d s^{\prime}$. Similarly, $f$ is linear and homogeneous with respect to $d r / d s^{\prime}$, $d^{2} r / d s d s^{\prime}$. Hence we have

$$
f=\psi(r) \frac{d r}{d s} \frac{d r}{d s^{\prime}}+2 \phi(r) \frac{d^{2} r}{d s d s^{\prime}},
$$

where $\psi(r)$ and $\phi(r)$ are functions of $r$.

These functions are determined by the second and third fundamental principles. The second gives $\psi(r)$ $=\phi^{\prime}(r)$, so that the problem is reduced to the determination of $\phi(r)$. This value of $\psi(r)$, however, permits $f$ to be written in the form

$$
2 d \mathrm{U} / d r \cdot d^{2} \mathrm{U} / d s d s^{\prime},
$$

where $U$ is a function of $r$ only, and

$$
d \mathrm{U} / d r=\sqrt{\phi(r)} \text {. }
$$

From this it is then shown that, if $\mathrm{T}$ be the so-called electrodynamic potential (electrokinetic energy) of the circuits-that is, the function the space variation of which, for any direction, is the force in that direction between the circuits -

$$
\mathrm{T}=\iint \frac{d \mathrm{U}}{d s} \frac{d \mathrm{U}}{d s^{\prime}} d s d s^{\prime},
$$

the currents being each unity, and the integrals being taken round the circuits.

The determination of $U$ is then effected by means of the third principle. It is first shown that $\mathrm{T}$ may be written as the integral of $\mathrm{F} d x+\mathrm{G} d y+\mathrm{H} d z$ round the circuit to which $d s$ belongs, $F$ denoting the integral round the other circuit of $U^{\prime 2} d s^{\prime} d r / d s^{\prime} .\left(x-x^{\prime}\right) / r$, and $\mathrm{G}, \mathrm{H}$ similar expressions. F, G, H are, in this theory, what Maxwell has called the components of vector potential. These values of $\mathrm{F}, \mathrm{G}, \mathrm{H}$, it is to be remarked, fulfil the relation

$$
d \mathrm{~F} / d x+d \mathrm{G} / d y+d \mathrm{H} / d z(=\mathrm{J})=0 .
$$

By applying the third principle it is proved that, if $\nabla^{2}$ have its ordinary signification, and $f^{\prime}(r)=U^{\prime 2} / r$, $\left(\mathrm{U}^{\prime}=d \mathrm{U} / d r\right), \nabla^{2} f(r)$ must be a constant, in order that the action of a closed non-varying solenoid on a complete circuit may be zero. Since $f(r)$ must be zero at infinity, this gives $f(r)=k / r$; and if the ordinary electromagnetic definition of unit current be taken, $k$ must be unity, so that $U^{\prime}= \pm I / \sqrt{ } r$. Hence the attraction between the elements is

$$
2 \gamma \gamma^{\prime} d s d s^{\prime} \frac{\mathbf{I}}{r^{2}}\left(\cos \epsilon-\frac{\beta}{2} \cos \theta \cos \theta^{\prime}\right),
$$

Ampère's well-known expression.

$$
\text { NO. I I 64, VOL. 45] }
$$

The above expressions for $\mathrm{F}, \mathrm{G}, \mathrm{H}$ reduce easily to $\int f(r) d x^{\prime}, \int f(r) d y^{\prime}, \int f(r) d z^{\prime}$, so that, putting in the value of $f(r)$, we get the well-known value of the mutual energy of the two circuits-

$$
\mathrm{T}=\iint \frac{\cos \epsilon}{r} d s d s^{\prime} .
$$

The theory of induction is next taken up. After a short discussion of some objections made by $\mathrm{M}$. Bertrand to the received method of deducing the laws of induction from the observed facts of electromagnetism, M. Poincaré proceeds to show that the electrokinetic energy of two currents is equal to the electrodynamic potential, and recalls Maxwell's application of Lagrange's dynamical equations to the theory of inductive action. $\mathrm{He}$ then deals at some length with the celebrated theory put forward by Weber for the action between two quantities, $e, e^{\prime}$, of electricity, as depending on their distance apart and their motion.

This we pass over, with the remark that Poincaré here discusses certain difficulties to which the theory leads in connection with the value it gives for the action between two current elements, and concludes with a short analysis of Maxwell's examination of the theory of induction as deduced from Weber's law. According to Maxwell ("El. and Mag.," vol. ii. p. 445, second edition), Weber's theory gives, for the inductive electromotive force exerted by the circuit in which the current $\gamma$ flows on the other, the equation-

$$
\mathrm{E}=\frac{d}{d t} \iint \gamma \frac{\mathbf{I}}{r} \frac{d r}{d s} \frac{d r}{d s^{\prime}} d s d s^{\prime},
$$

which, for a closed circuit, agrees with the well-known equation-

$$
\mathrm{E}=-\frac{d}{d t} \iint \gamma \frac{\cos \epsilon}{r} d s d s
$$

M. Poincaré points out that this apparent agreement of the two theories is due to the fact that Maxwell has overlooked certain terms which contribute to the value of $\mathrm{E}$, and which do not give a zero result when integrated round a closed circuit.

The expressions given by Weber and Neumann for the mutual potential of two current elements are next considered, and shown to be included in the general expression given for the same potential by Helmholtz. By means of this expression Helmholtz's general electrodynamic theory is introduced, and then follows an elaborate comparison of the theories of Helmholtz and Maxwell. It is shown that Helmholtz's theory leads to the value of $\mathrm{T}$ for conduction in three dimensions given by the equation-

$$
\mathrm{T}=\frac{1}{2} \int(\mathrm{F} u+\mathrm{G} v+\mathrm{H} w) d \widetilde{\omega},
$$

where $d \widetilde{w}$ is an element of volume, $u, v, w$ the components of currents, and the integral is extended throughout all space. F, G, H, are, of course, the components of vector potential, and in this theory are given by equations-

$$
\begin{gathered}
\mathrm{F}=\int \gamma^{\prime} d x^{\prime} / r+\frac{1}{2}(\mathbf{1}-k) d \psi / d x, \& \mathrm{c} . \\
\psi=\int \gamma^{\prime} d s^{\prime} \cdot d r / d s^{\prime} .
\end{gathered}
$$

If $\rho$ be the density of free electricity at any point,

$$
d u / d x+d v / d y+d z v / d z=-d p / d t,
$$

and this, when instead of $\gamma^{\prime} d r / d s^{\prime}$ is substituted its value in terms of $u^{\prime}, v^{\prime}, v^{\prime}$, gives, by an application of Green's theorem, the result-

$$
\psi=\int r d \rho^{\prime} / d t \cdot d \widetilde{\omega}^{\prime},
$$

where $d \widetilde{\omega}^{\prime}$ is an element of the space in which the current 
$\gamma^{\prime}$ is flowing. Now, if in the insulating dielectric two like quantities of electricity, $e, e^{\prime}$, would produce a repulsive force of magnitude $e e^{t} / \lambda r^{2}$, the electrostatic potential $\phi$, due to the free electricity of density $\rho^{\prime}$, is given by the equation-

$$
\lambda \phi=\int \rho^{\prime} d \widetilde{\omega}^{\prime} / r .
$$

Thus, from the foregoing values of $\mathrm{F}, \mathrm{G}, \mathrm{H}$, three equations are obtained, viz.,

$$
\nabla^{2} \mathrm{~F}=:-4 \pi u+(\mathbf{I}-k) \lambda d^{2} \phi / d x d t,
$$

with two similar equations for $\nabla^{2} \mathrm{G}$ and $\nabla^{2} \mathrm{H}$. These give

$$
a \mathrm{~F} / d x+d \mathrm{G} / d y+d \mathrm{H} / d z=-k \lambda d \phi / d t
$$

which is zero if $k$ or $\lambda$ be zero.

If, now, the magnetic inductive capacity be taken for the moment as unty, and the magnetic force $(a, \beta, \gamma)$ be so defined that $\Gamma$ is $\gamma$ times the magnetic induction through the circuir in which the current whose numerical magnitude is $\gamma$ flows, we deduce easily the equations-

so that there follow

$$
\alpha=d \mathrm{H} / d y-d \mathrm{G} / d z, \& c \text {; }
$$

$$
\begin{aligned}
& \frac{d \gamma}{d \nu}-\frac{d \beta}{d z}=4 \pi u-\lambda \frac{d^{2} \phi}{d x} \frac{d t}{d t}, \\
& \text { \&c., \&c. }
\end{aligned}
$$

These coincide with the equations of currents given by Maxwell when the last terms are omitted. We must therefore either put $\lambda=0$, or (since $d \phi / d t$ is not in general zero) $\phi=0$ to reduce to Maxwell's theory.

Poincaré next proves that unless the $k$ in Helmhoitz's theory be not less than zero, the sum of the electrostatic and electrokinetic energies may diminish indefinitely from an infinitely small value, so that there would be unstable equilibrium. This affords another reason for rejecting the theory of Weber, in which $k=-1$.

In chapter v. Poincaré passes from the theory of Helmholtz to that of Maxwell. He first considers magnetic and dielectric polatrization according to a modified and corrected version of the theory of Poisson. He supposes the dielectric space filled with conducting particles separated by other material, the dielectric proper, which completely insulates these bodies from one another. These conducting bodies are supposed to be electrically polarized, so that electric displacement $(f, g, h)$ is set up in the medium. A farallel theory of magnetic polarization is considered, and the electric displacement is simply the electric analogue of the intensity of magnetization of the medium-that is, the magnetic moment at each point per unit of volume. If $\epsilon$ be the ratio of the volume occupied by the conducting particles to the whole volume of the dielectric space in which they are embedded, the specific inductive capacity, $\mathrm{K}$, of the medium is found to be $\lambda \phi(\epsilon)$, where $\phi(\epsilon)$ is a furiction analogous to $(I+2 \epsilon) /(I-\epsilon)$ (the corresponding function for the case in which the conducting particles are spheres) in that it becomes infinite when $\epsilon=\mathrm{I}$.

According to Poincaré, $\lambda$ is the specific inductive capacity of the clielectric medium proper, or insulator between these conducting bodies, and is very small. In order, therefore, that $\mathrm{K}$ may be finite, it is necessary that $\epsilon$ may be very nearly equal to unity.

The electrostatic potential, $\phi$, at any point is that due to the free electricity present on conductors, and to the electricity developed throughout the medium by its polarization. The electrification, in fact, consists of two parts - a volume censity on the dielectric depending on the electric displacement, and of amount $-(d f / d x+$ $d \sigma / d y+d h / d z)$, and a resultant surface density, $\sigma^{\prime}=\sigma-$ $($ lf $+m g+n h)$, where $\sigma$ is the surface density of the electricity present in the form of charges on conductors, and $l f+m g+n i$ (in which $l, m, n$ are the direction cosines of the normal to the surface directed inwards towards the dielectric) is the surface distribution due to the polarization. By speaking of this as the electrification, what is meant is that this electrification existing in the dielectric medium proper would give the observed potential, $\phi$, at each point. Thus

$$
\phi=\frac{\mathbf{I}}{\lambda}\left\{\int \frac{d \widetilde{\omega}}{\rho}+\int \sigma^{\prime} \frac{d \mathrm{~S}}{r}\right\} .
$$

The value of the electrostatic energy, $U$, according to this dielectric theory, is given by

$$
\mathrm{U}=\frac{\lambda}{8 \pi} \int \mathrm{\Sigma}\left(\frac{d \phi}{d x}\right)^{2} d \widetilde{\omega}+\frac{2 \pi}{\mathrm{K}-\lambda} \int \mathrm{\Sigma} f^{2} d \widetilde{\omega}
$$

If there are applied electromotive forces, $\mathrm{X}, \mathrm{X}, \mathrm{Z}$, their values are given by

$$
\mathrm{X}=-d \phi / d x-4 \pi f /(\mathrm{K}-\lambda),
$$

with similar equations for $\mathrm{Y}$ and $\mathrm{Z}$. Hence, if $\mathrm{X}, \mathrm{Y}, \mathrm{Z}=\mathrm{0}$ -that is, if there is nothing but electrostatic action-the electrostatic energy is given by

$$
\mathrm{U}=\int \frac{\mathrm{K}}{8 \pi} \mathrm{\Sigma}\left(\frac{d \phi}{d x}\right)^{2} d \widetilde{\omega} .
$$

If $\mathrm{X}, \mathrm{Y}, \mathrm{Z}$ are not zero, the electrostatic energy becomes $2 \pi / K . \int \Sigma\left(f^{2}\right) d \widetilde{\omega}$ (which is Maxwell's expression), provided $\lambda=0$. We have to inquire what reasons can be adduced for putting $\lambda=0$ in this theory.

M. Poincaré shows that the velocity of propagation of a wave of longitudinal displacement in Helmholtz's theory

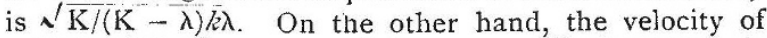
propagation of a wave of transverse displacement is $\sqrt{\mathrm{I} / \mu(\mathrm{K}-\lambda)}$. Thus there will be no longitudinal wave if one of the conditions, $k=0, \lambda=0, K=\lambda$, hold. The last condition would make the velocity of propagation of waves of transverse displacement infinite, and must be rejected for every medium, even so-called vacuum, in which light is propagated. Poincaré adopts the second hypothesis.

Further, if to $\lambda$ a positive value sensibly greater than zero be assigned, a wave of transverse displacement will be given a velocity sensibly greater than that of light. Thus, to pass to Maxwell's theory, it is necessary to make $\lambda$ insensible, as this gives for the velocity of waves of transverse displacement his value $I / \sqrt{\mu \mathrm{K}}$, which is known by experiment to be the velocity of light.

The adoption of this value of $\lambda$ leads to all the electromagnetic equations of Maxwell, and to the conditions $J=0, d u / d x+d v / d y+d w / d z=0$, the last of which expresses that electricity considered as the analogue of a fluid is incompressible--that is, that all currents flowing are closed currents.

It may be pointed out here that this conclusion agrees with that arrived at by Mr. R. T. Glazebrook in his comparison of Maxwell's electromagnetic equations with those of Helmholtz and Lorentz, and in his further paper on the general equations of the electromagnetic field (Proc. Camb. Phil. Soc., vol. v., Part ii., 1884). Mr. Glazebrook's result is that $\Phi$, the electrostatic potential of Helmholtz's paper must be zero everywhere in order to pass from Helmholtz's theory to that of Maxwell. But $\Phi$ is what Poincaré has denoted by $\lambda \phi$, so that the conditions are the same.

It has been attempted to make the transition to Maxwell's theory by putting $k=0$. This would not suffice alone, as Poincaré points out. For, while giving at once $\mathrm{J}=0$, it would fail to give Maxwell's velocity for transverse waves unless further $\lambda=0$, which by itself would suffice to effect the transition irrespective of the value of $k$.

Poincare thus supposes, in opposition to the older dielectric theories, that even vacuum, or the ether of the inter-planetary spaces, consists of polarizable conducting matter embedded in an insulating medium of infinitely small inductive capacity, $\lambda$. According to

NO. I I 64 , VOL. 45] 
Mossotti's theory, which is the starting-point of all mathematical theories of polarization, the conducting particles are spherical, and therefore, if $\epsilon$ be the ratio of the volume of the spheres to the total volume of the medium, the value of $\mathrm{K}$ is $(I+2 \epsilon) /(I-e)$. It is here assumed that the specific inductive capacity of the insulating dielectric is unity. Poincaré, however, sees no reason for making this particular assumption, and takes it as $\lambda$, a quantity which, if Maxwell's theory be the true one, must be exceedingly small. This involves, as already stated, $K=\lambda \phi(\epsilon)$, where $\phi(\epsilon)$ is a quantity which becomes very great when $\epsilon=\mathrm{I}$. Thus, according to Maxwell's theory, the conducting particles are separated by infinitely thin insulating partitions, so that they practically fill the whole space. Of course, the physical fact may be very different from that here supposed: the theory only furnishes a picture, not perhaps altogether clear and intelligible, of the structure of the medium and its functions.

It may be said that the infinitely small inductive capacity, $\lambda$, of the medium, itself requires physical explanation. This is quite true ; but so also does the specific inductive capacity equal to unity assumed for vacuum or air in the ordinary theories. In fact, such dielectric theories as have been put forward, involving merely polarization of the medium, only give an explanation of the difference between the electric behaviour of one medium and another, and furnish none whatever of the real rationale of the propagation of electric action.

That the value of $\phi$ may be finite, it is necessary that the values of the volume density, $\rho$, and the surface density, $\sigma^{\prime}$, may be infinitely small, since

$$
\phi=\int \frac{\rho}{\lambda r} d \widetilde{\omega}+\int \frac{\sigma}{\lambda r} d \mathrm{~S} .
$$

Here $\rho$ is the volume density due to the surface distributions on the opposite faces of the partitions between the conducting particles, and this, it is easy to see, will be infinitely small. Also, $\sigma^{\prime}$ is the sum of the actual density (surface density of charge) on the surface of the conductors, and the density, which is the surface manifestation of the polarization of the conducting particles, or $\sigma^{\prime}=\sigma-(l f+m g+n h)$. This also can be conceived as exceedingly small, so that $\dot{\phi}$ may have a finite value.

Further reasons for preferring the theory of Maxwell are discussed in chapter vi., which is entitled "The Unity of Electric Force." "This chapter consists of an exposition of Hertz's modification of Maxwell's electromagnetic theory-a modification, it is to be remarked, practically given also, but in vector form, by $\mathrm{Mr}$ Oliver Heaviside, in various papers in the Philosophical Magazine. When made, it exhibits a striking parallelism between the equations of electric and magnetic force, and leads to some remarkable theorems. Using Maxwell's equations, and deviating slightly from Poincarés mode of presenting the equations, we have, if $k$ now denote electric conductivity of the medium, and $\mathrm{P}, \mathrm{Q}, \mathrm{R}$ components of electric force-

$$
\left(k+\frac{\mathrm{K}}{4 \pi} \frac{d}{d t}\right) \mathrm{P}=\frac{\mathrm{I}}{4 \pi}\left(\frac{d \gamma}{d y}-\frac{d \beta}{d z}\right),
$$

with two similar equations for $Q$ and $R$. But also we have-

$$
\frac{\mu}{4 \pi} \frac{d \alpha}{d t}=-\frac{\mathbf{I}}{4 \pi}\left(\frac{d \mathrm{R}}{d y}-\frac{d \mathrm{Q}}{d z}\right)
$$

with two similar equations for $\beta$ and $\gamma$. These last may, by the introduction of a non-existent quantity, $g$, be written-

$$
\left(s+\frac{\mu}{4 \pi} \frac{d}{d t}\right) \alpha=-\frac{\mathrm{I}}{4 \pi}\left(\frac{d \mathrm{R}}{d y}-\frac{d \mathrm{Q}}{d z}\right)
$$$$
\text { \&c., \&c. }
$$

The quantity $g$, Heaviside points out, is the proper magnetic analogue to $k$, and may therefore be called the magnetic conductivity. Its reciprocal would be the No. I I64, VOL. 45$]$ true magnetic resistivity of the medium. Of course, in an insulating medium $k$ is also zero.

According to Maxwell's theory, P, Q, R, $a, \beta, \gamma$ fulfil the equations-

$$
\begin{aligned}
& \frac{d \mathrm{P}}{d x}+\frac{d \mathrm{Q}}{d y}+\frac{d \mathrm{R}}{d z}=0, \\
& \frac{d \alpha}{d x}+\frac{d \beta}{d y}+\frac{d \gamma}{d z}=0 ;
\end{aligned}
$$

the first, since $d u / d x+d v / d y+d v / d z=0$, and the second because the magnetic force in the medium, being supposed purely inductive, must fulfil the solenoidal condition, except at the (vortex) origin of the disturbance.

There is therefore, in Maxwell's theory, a perfect reciprocity of relation between the electric and magnetic quantities. Hence we might infer, from the magnetic phenomena following from electric currents or flow of electricity, an analugous set of electric phenomena following from the flow of magnetism. Now we know that if a magnet varies in strength it produces an electromotive force of components $\mathrm{P}, \mathrm{Q}, \mathrm{R}$, at every point of the surrounding space. This we may suppose due to a current of magnetism flowing from one end of the magnet to the other, and thus producing the variation in the magnet's strength. The directions of the components of electric force at any point are in fact coincident with those of the components of vector potential produced by the magnet at that point, and are equal to the time-rates of variation of these components. But why should this not be regarded as an electrostatic field in the ordinary sense of the term? For example, a current of electricity, flowing round a closed circuit, produces a magnetic field equivalent to that which would be produced by a magnetic shell of proper strength, and having its edge coincident with the circuit. Of this current a closed solenoid varying continuously in magnetic strength (for example, a closed solenoid in which the magnetizing current is varying in strength) is the magnetic analogue, and ought in the same way to be equivalent to an electric shell, in the sense of producing an identical electric field. Such a shell ought to be subject in an electric field to dynamical action; and further, two such varying solenoids ought to exert the same mutual dynamical action, as would the two equivalent electric shells if placed in the same configuration. The second of these conclusions asserts that the dynamical action on such a shell depends only on the electric field in which it is placed, and that its action on the other varying solenoid is due to its producing exactly the same electric field as the equivalent electric shell would produce. This is what Poincaré gives as Hertz's principle of the unity of electric force.

Of course, it is to be noticed that the second conclusion does not follow from the first. We cannot reason that because the mutual action of an electric shell and a varying solenoid is the same as that of two electric shells, therefore the mutual action of two solenoids is the same as that of two electric shells.

If, however, we assume that the dynamical action on a closed varying solenoid depends only on the electric field in which it is placed, we can say that the mutual action of two varying solenoids is the same as that of their equivalent electric shells.

M. Poincaré calculates the work done in effecting a relative displacement of two such varying solenoids, and finds that it is equal to the change in the electrostatic energy of the system, as the change in the electrokinetic energy is all accounted for otherwise. Now, the electrostatic energy of a system is given as we have seen by the equation-

where

$$
\mathrm{U}=\int\left\{\frac{\lambda}{8 \pi} \mathrm{\Sigma}\left(\frac{d \phi}{d x}\right)^{2}+\frac{2 \pi}{\mathrm{K}-\lambda} \mathrm{\Sigma}\left(f^{2}\right)\right\} d \widetilde{\omega},
$$

$$
f=-(\mathrm{K}-\lambda) / 4 \pi \cdot d \mathrm{~F} / d t+i \phi / d x \text {. }
$$


This may be written

$$
\mathrm{U}=\int\left\{\frac{\lambda}{8 \pi} \Sigma\left(\frac{d \phi}{d x}\right)^{2}+\frac{\mathrm{K}-\lambda}{8 \pi} \Sigma\left(\frac{d \phi}{d x}+\frac{d \mathrm{~F}}{d t}\right)^{2}\right\} d \boldsymbol{\omega} .
$$

Now, if there be no inductive action-that is, if the field be wholly electrostatic-

$$
d \mathrm{~F} / d t, d \mathrm{G} / d t, d \mathrm{H} / d t=0
$$

and hence, for two electric shells-

$$
\mathrm{U}=\int \frac{\mathrm{K}}{8 \pi} \Sigma\left(\frac{d \phi}{d x}\right)^{2} d \widetilde{\omega} .
$$

On the other hand, if the action be wholly inductivethat is, if we have no so-called electrostatic action--

$$
d \phi / d x, d \phi / d y, d \phi / d z=0,
$$

and for two varying solenoids we have-

$$
\mathrm{U}^{\prime}=\int \frac{\mathrm{K}-\lambda}{8 \pi} \mathrm{\Sigma}\left(\frac{d \mathrm{~F}}{d t}\right)^{2} d \widetilde{\omega}
$$

If, then, there be the same mutual action between the two varying solenoids as between their equivalent electric shells, we must have $U=U^{\prime}$. But because of the equivalence the value of $d \mathrm{~F} / d t$, \&c., produced at any point by either solenoid, must be the same as those of $d \phi / d x$, \&c., produced at the same point when the solenoid is replaced by its equivalent electric shell. Thus we get $\mathrm{U} / \mathrm{U}^{\prime}=\mathrm{K} /(\mathrm{K}-\lambda)$, and therefore $\lambda=0$. Thus, if the principle of the unity of electric force is true, $\lambda=0$, and we have Maxwell's theory.

Dr. Oliver Lodge has, as is well known, endeavoured to detect the existence of an electrostatic field produced by varying magnetic action (NATURE, May 23, I 889 , and Electrician, May I7, I889), and has reason to believe that he has been successful. It is also possible, as Poincaré suggests, that this kind of electrostatic action may be developed when iron rings, \&c., are placed in the field of an alternating electromagnet, as in the experiments of Elihu Thomson.

In a note, which forms a supplement to the comparison of the theories of Helmholtz and Maxwell, M. Poincaré points out that when the mutual action of a varying solenoid and an electric shell is considered, contradictory results are obtained according as the solenoid is regarded as fixed and the shell movable, or the shell fixed and the solenoid movable. Thus the theory of Helmholtz in this application does not give fulfilment of the third law of motion.

Possibly, some such theory as this may throw some light on the electric phenomena of voltaic cells, with their finite steps of potential across the surfaces of separation of dissimilar substances, and help to refer the production of all currents to the single cause-electromagnetic action.

We come now to the discussion which the book contains of the experiments of Hertz. This fills considerably more than one-half of the work, and we cannot, in the space left at our disposal, give an adequate account of it. Of the experiments themselves it is not necessary to say anything, as they have been fully and ably discussed in NATURE by Mr. Trouton (February 21, I889). Hertz's own theory of the radiation of electric and magnetic energy has also been given in these pages by Dr. Lodge (February 21, 1839, et seq.). Poincarés presentment of the theory is, however, marked by many points of originality, and abounds in acute and interesting remarks.

The theory of the dumb-bell exciter used by Hertz is first considered, then the field produced is discussed, and, last of all, the action of the resonator or receiver is dealt with. Taking the exciter as a couple of spheres, $15 \mathrm{cms}$. in radius, placed with their centres $150 \mathrm{cms}$. apart, and joined by a wire $\frac{1}{2} \mathrm{~cm}$. in diameter, Poincaré calculates (I) the capacity, (2) the self-induction of the arrangement. The value of the capacity of the arrangement of two spheres used by Hertz in the calculation of the period of the exciter was that of each ' of the spheres by itself, viz. I $5 \mathrm{cms}$. Now, if one of the spheres were alone in its own field with a charge $q$ and at a potential $\mathrm{V}$, we should have $q / \mathrm{V}=15$. But at any instant when the charge of one sphere is $q$, that of the other sphere is $-q$; and since the spheres may be taken as nearly without mutual influence, the difference of potential between them is $2 \mathrm{~V}$. The capacity is, then, $q / 2 \mathrm{~V}$, or $7.5 \mathrm{cms}$. - half the value used by Hertz. That this is the proper value to use for the capacity is easily verified by a reference to the mode of establishing the equation of oscillation, when it is seen that the capacity is really defined by that equation as the charge on one of the spheres divided by their difference of potential.

The calculation of the self-induction given by Poincaré is interesting. Regarding, as an approximation, the currents in the spheres, and the influence of the sparkinterval, as negligible, and taking the wire as of length $l$ (equal to the distance between the centres of the bulbs), and of diameter $d$, and assuming that the current is wholly on the surface of the wire (which it is approximately, when in rapid alternation) he finds-

$$
\mathrm{L}=2 l\left\{\log \frac{4 l}{d}-\mathbf{I}+\frac{k-\mathbf{I}}{2}\right\},
$$

where $k$ is the quantity which appears in Helmholtz's theory.

This differs from the value given by Hertz in having - I for the middle term within the brackets instead of - 75 , and $(k-1) / 2$ instead of $(\mathbf{I}-k) / 2$ for the third term. The first discrepancy arises through the currents having been taken by Hertz as uniform over the crosssection of the wire, and the second probably through an error in sign. The self-induction, $\mathrm{L}$, is $1902 \mathrm{cms}$. if the term involving $k$ is not taken into account, and (1902 + I 50$) \mathrm{cms}$. if $k$ be put equal to zero.

Calculating the period $\mathrm{T}(=2 \pi \sqrt{\mathrm{LC}}$, where $\mathrm{C}$ is the capacity in electromagnetic units), we find it to be $2.5 \mathrm{I} \times \mathrm{IO}^{-8}$ seconds, and multiplying by the ratio of the electromagnesic unit of quantity to the electrostatic unit, or $v$, we get for the wave-length 7.53 metres. Hertz gives I.77 for the calculated half period, and 5.31 for the corresponding half wave-length. On account of the error in the estimation of the capacity, it is clear that this value of the half period and half wave-length must be divided by $\sqrt{2}$, and this brings them into agreement with the values first stated. There is, however, a serious discrepancy between the results of theory and experiment, which we shall notice presently.

The calculation of period, \&c., of course proceeds on the assumption that the resistance is negligible, and this is no doubt the case to a sufficient degree of approximation. In the theory itself, also, no account is taken of the induction coil or of the displacement currents in the dielectric; further, the energy is dissipated, not merely by the production of heat, but by radiation into the dielectric. That the influence of the induction coil is indeed negligible Poincaré gives reasons for supposing; in fact, on account of the enormous self-induction of the induction bobbin, and the small mutual induction of the exciter and the bobbin, the corrected differential equation is the one formerly found for the oscillation, with the addition of an exceedingly small term, so that the solution is practically the same as before. (Here, p. 162, the expression " $a$ étant très grand" should be " $a$ étant très petit"). As Poincaré states, the vibration of the exciter is like that of a very small pendulum attached to a massive pendulum of long period; the period of the former is very little affected by its mode of support. For a similar reason the period is very little affected by the very considerable capacity of the bobbin.

Experimenting on the velocity of propagation of electro-

NO. I 164 , VOL. 45] 
magnetic waves, Hertz found that the half wave-length in air was about 4.5 metres, the corrected period of the vibrator used being about $2 \times 10^{-8}$ seconds. This gives a velocity of propagation of $900 /\left(2 \times 10^{-8}\right)\left(\right.$ or $\left.4.5 \times 10^{10}\right)$ $\mathrm{cms}$. per second, exceeding the velocity of light by about 50 per cent. For the wave-length in wires, however, he found a value which gives a velocity of propagation nearly equal to the velocity of light, when the correction of the period for error in capacity is taken into account. (Later, M. Poincaré gives the half wave-length in air for these experiments as 4.8 metres.)

Herr Lechner, experimenting at Vienna, has also found a velocity of propagation in wires very approximately equal to the velocity of light, which thus confirms Hertz's result. On the other hand, MM. Sarasin and de la Rive, experimenting at Geneva in 1890 , found that the wavelength observed depends very much on the dimensions of the resonator. But using an exciter exactly similar to that of Hertz, and of the same dimensions, and a resona. tor $75 \mathrm{cms}$. in diameter, and therefore nearly an exact copy of that employed by Hertz, they found a half wavelength of 3 metres, instead of 4.8 metres as found by Hertz. Thus there is a discrepancy between the two results which it is difficult to explain. Hertz himself gives a possible explanation, in a letter to M. Poincaré which is quoted in a note on some recent experiments which is printed as an appendix. On account of its interest, we take the liberty here of translating the extract quoted. It is to be noted that what is called the wavelength here is the distance from node to node, or half the complete wave-length.

"It is difficult for me to believe that I have been misled in the second method into finding 4.8 metres instead of 3 metres; but since the result of Messrs. Sarasin and de la Rive has every theoretical appearance of truth, I have endeavoured to find out the cause of the difference. Here are two ways of explaining it. The waves were produced between two parallel walls of a room, and I have taken account of the reflective action of only one of them. Let us suppose, to begin with, that the length of the room is an exact multiple of the wave-length, say three wave-lengths. We shall have two well-marked nodes at the exact distance. If the length of the room is four wave-lengths, we shall have three well-marked nodes. But if the length of the room is intermediate between these, and nearer the former than the latter, we shall have two less distinct nodes at a distance apart greater than a wave-length. This explanation would appear to me satisfactory, if the difference were not too great.

"The other way of explaining the difference is this. My reflecting plate of zinc was fixed in a niche in the wall, and it is possible that the projecting parts of the wall may have had the effect of carrying off the nodes to a greater distance from the wall, and thus of giving too great an apparent length as measured. But it is also true that the niche was from 5 to 6 metres in width, and it does not seem to me very probable that it can have had any great effect. ${ }^{1}$

"I therefore cannot tell precisely the cause of my error; but $I$ believe there must be some way of explaining it For a long time I have sought in vain to find a probable cause for the difference of velocity in air and in wires; and I had myself found, before Messrs. Sarasin and de la Rive, that there is no difference for short waves of $30 \mathrm{cms}$. in length. The results of these gentlemen, however, give the same velocity for long waves, and contradict my experiments"

Connected with this point M. Poincaré has some instructive remarks on what Messrs. Sarasin and de la Rive have observed and called multiple resonance, and which

'For an interesting discussion of the effects of reflecting plates of different dimensions, see a paper by Mr. Trouton in the Phil. Mag., July rigi.

NO. I I 64, VOL. 45] has also been observed by Fitzgerald and Trouton. Their supposition is that the exciter gives rise neither to a single vibration of distinct period, nor to a limited number of distinct vibrations, but rather to such a complex of vibrations as would give a wide band of continuous spectrum. Thus all vibrations, agreeing with possible modes of vibration of the resonator would be reinforced. That this explanation is not borne out by the theory is true, but on account of the incompleteness of the theory it is not possible to attach much weight to this fact. It is hard to believe that the vibrations can be perfectly simple.

Poincaré proposes, however, the following explanation. For various reasons, he thinks the logarithmic decrement of the vibrations of the exciter is probably much greater than that of the resonator, and so the ribrations of the exciter diminish in amplitude more quickly than those which by any cause are set up in the resonator. Thus the resonator, being started by the exciter, would continue its vibrations after those of the exciter had become insensible, but would then vibrate in its own proper period, thus giving vibrations of longer period and of greater wave-length than those which excited it. The wavelength, being determined by interference, and used with the too short period of the exciter, would of course give too great a velocity of propagation. With this explanation Hertz has expressed himself as practically in accord and so a possible way out of the difficulty seems opened up. As Hertz remarks, the oscillations of the exciter, represented graphically, do not give a curve of sines pure and simple, but a curve of sines the amplitude of which gradually diminishes. Such an oscillation will cause all resonators receiving it to vibrate, but those in tune with the exciter more violently than the otkers. A mathematical investigation of the point is given by M. Poincaré, which explains the result, shown by experiment, that the apparent spectrum found by Sarasin and de la Rive seems more extended when wires are connected to the vibrator, than when the propagation takes place freely in air.

Whether this explanation be satisfactory or not, there can be no doubt, on the whole, that the electromagnetic theory of light is substantially true. The theory is far from complete, and there are many outstanding points which require further theoretical and experimental elucidation. Some of these are touched on by Poincaré in his discussion of the field produced by exciters of different forms, and the theory of the resonator, but especially in a valuable series of notes which he has added to his lectures. These deal with special topics, which are there treated with more detail than was possible in the body of the work. Such, for example, are his notes on multiple resonance, the calculation of the period, and the propagation of waves in sinuous wires.

This article has run to too great a length, and must here close. M. Poincaré's work ought to be read by everyone interested in Maxwell's great scientific generalization-the greatest, perhaps, ever made by a natural philosopher since the days of Newton-and in its remarkable experimental verification by Hertz. There never was, perhaps, a time of greater mathematical and physical activity than the present, but withal it is marked by a care for the scientific student which no previous age ever displayed. It is no small encouragement to humbler scientific workers when masters of analysis like $M$. Poincaré take the trouble to publish, in a connected form, their lectures and researches on the current scientific questions of the day. Besides earning the gratitude of those who are thus admitted within the circle of their pupils, by immediately communicating their discoveries and expositions in this manner to the general scientific public, they multiply many-fold the direct effect of their work on scientific progress.

A. Gray. 